\title{
Genetic Susceptibility to Esophageal Cancer due to CYP1A1 Gene Variant rs4646903 in Tobacco Addicted Patients of Pashtun Ethnicity: a Case Control Study in Khyber Pakhtunkhwa Province of Pakistan
}

\author{
Zakiullah$^{1}$, Muhammad Saeed ${ }^{1 *}$,Sajid Ali², Nabila Javed ${ }^{3}$, Muhammad \\ Khisroon $^{4}$, Basir Muhammad ${ }^{5}$, Fazli Khuda ${ }^{1}$, Saeed Ahmad ${ }^{6}$, Mohammad Ismail ${ }^{1}$
}

\begin{abstract}
The purpose of this study was to evaluate associations of the CYP1Al gene variant rs4646903 polymorphism with the risk of developing esophageal cancer (EC). A case-control study was carried out in Pashtun population of Khyber Pakhtunkhwa province of Pakistan in which 140 hospital based EC cases and 196 population based healthy controls exposed to similar environmental conditions were included. A specific method based on the real time polymerase chain reaction (RT-PCR) was used to detect genotypes in case and control groups and results were then analyzed with SPSS version 20. In our population, individuals with CC and TC genotypes of the CYP1A1 rs4646903 polymorphism had significantly higher risk of EC (adjusted odds (OR): 15.709,95\% CI: 6.065-40.686, OR: $3.25695 \% \mathrm{CI}: 1.902-5.574$ respectively). The ' $\mathrm{C}$ ' allele was strongly associated with the disease $(\mathbf{p}<0.0001)$. Adjusted OR was higher $(1.5$ times in $\mathrm{C} / \mathrm{C}$ ) in case of variant alleles that show the contribution of environmental and nutritional factors towards the development of EC. Our findings suggest that presence of the ' $C$ ' allele of rs4646903 (T>C) may be one of the risk alleles for EC susceptibility in Pashtun population.
\end{abstract}

Keywords: Esophageal cancer risk - CYP1A1 gene variant rs4646903 - Pashtun population - Pakistan

Asian Pac J Cancer Prev, 15 (16), 6715-6720

\section{Introduction}

Esophageal cancer (EC) is the 8th most common cancer in the world and 6th leading cause of death due to cancer worldwide (Mao et al., 2012; Weng et al., 2012). Majority of the new EC cases are diagnosed at stage III/IV, with a poor 5-year survival prognosis. In United States, 14,280 out of total 16,470 new EC cases reported in 2008 died of this disease (Jemal et al., 2011). In this scenario, experts in the field of EC have internationally recognized the fact that for the development of any preventive strategies, it is imperative to elucidate the causes and risk factors responsible for the epidemiology of EC (Eslick et al. 2009; Mao et al., 2012). EC involves the malignancies that arise from the epithelial surface of the esophagus, with two major subtypes i.e., Esophageal Squamous Cell carcinoma (ESCC) and Esophageal Adenocarcinoma (EADC). ESCC primarily arises in the upper part of the esophagus, while EADC arises mainly from the glandular cells near the junction of the esophagus and stomach. ESCC has similarity with head and neck cancer in their appearance and association with tobacco and alcohol intake, while EADC is most commonly associated with a history of gastro esophageal reflux disease(GERD) and Barrett's esophagitis (Kollarova et al., 2007). Although it is found throughout the world but majority of the cases, especially ESCC, occur in developing counties with striking geographic variations.

A very high incidence rate has been reported in SouthCentral Asia that is also known as "Asian cancer belt" that stretches eastwards from Iran through Turkmenistan, Afghanistan, Uzbekistan, Kazakhstan into Northern China and Mongolia with annual age-standardized incidence rate (ASIR) as high as 100 new cases per 100,000 population and SCC as the most common subtype (Badar et al., 2005; Weng et al., 2012). In Pakistan, regions adjacent to the cancer built extending from Quetta through Dera Ismail Khan into Khyber Pakhtunkhwa have high incidence rate as compared to other areas and some expert suggest it as the 'extension of the Asian cancer belt' (Figure 1) (Jamal, 2010). Many factors have been implicated in these areas in the etiology of the disease including environmental factors

${ }^{1}$ Department of Pharmacy, Faculty of Life \& Environmental Sciences, ${ }^{2}$ Centre of Biotechnology \& Microbiology, Faculty of Life \& Environmental Sciences, University of Peshawar, ${ }^{3}$ Institute of Radiotherapy \& Nuclear Medicine (IRNUM), ${ }^{4}$ Department of Zoology, Faculty of Life \& Environmental Sciences, University of Peshawar, ${ }^{5}$ Department of Clinical Oncology, BINOR Cancer Hospital, Bannu, ${ }^{6}$ Department of Microbiology \& Biotechnology, Faculty of Life \& Environmental Sciences, Sarhad University of Science \& Information Technology, Peshawar Khyber Pakhtunkhwa, Pakistan *For correspondence: saeedrph@upesh.edu.pk 
such as mineral contents of the soil; dietary factors such as diet low in fruits, vegetables and animal proteins; personal habits such as tobacco intake and alcoholism (Cortes and Villasenor, 1997; Sharma et al., 2011; Yi and Li 2012). Besides that, age, gender, poor socioeconomic status and genetic factors also contribute towards the disease (Mao et al., 2012; Amani et al., 2013).

Studies conducted in Pakistan have reported tobacco use including cigarette smoking, naswar intake and paan chewing as the main risk factors for EC (OR: 3.3 and 2 for cigarette and naswar respectively) (Jamal et al., 1997; Afridi et al., 2000). The confirmed carcinogenic species reported in tobacco mainly include nitrosamines, aromatic amines and polyaromatic hydrocarbons (PAH) (Yun et al., 2013). Tobacco use and alcoholism have also been shown to possess synergistic effects increasing the relative risk over 100-times than that of induced by tobacco or alcohol use alone. This is owing to the reason that fat soluble compounds are more soluble in ethanol and are thus easily absorbed (Blot et al., 2006; Mao et al., 2012). More than 20 different nitrosamines have been shown to play an important role in inducing EC in animals displaying time-and dose-dependency (Castellsague et al., 1999).

These compounds are actually pro-carcinogens and are metabolized by a family of ubiquitous enzymes Cytochrome P450 (CYPs).Various isoforms of CYPs including $C Y P 1 A l$ are reportedly involved in activation process (D'Errico et al., 1996; Olshan et al., 2000). However, the expression of these enzymes differs in individuals resulting in differences in the metabolic processing of carcinogens. Certain individuals are genetically more susceptible to cancer when exposed to carcinogens owing to their genotype for enzymes responsible for their activation or detoxification (Abbas et al., 2014). This is evident from the finding that only a small number of individuals among those exposed to tobacco or alcohol under the same environmental conditions develop cancer in their life time. CYPIAl metabolically converts majority of tobacco containing pro-carcinogens into reactive metabolites that have carcinogenic effects (Taskiran et al., 2006; Abbas et al., 2014). Allelic variants influencing the enzyme activity of $C Y P 1 A 1$ along with environmental factors such as tobacco use play key roles in making individuals susceptible to different types of cancers (Xia et al., 2013). The CYPIAl gene located on chromosome 15q22-q24 encodes an enzyme with aryl hydrocarbon hydroxylase activity that plays important role in the metabolism of polycyclic aromatic hydrocarbons (PAH) and nitrosamines from tobacco, and inherited differences in metabolic capacity are considered to play a major role in carcinogenesis (Sabitha et al., 2010). Certain allelic variations in the CYPIAl gene and prolonged exposure to tobacco products could lead to higher levels of reactive metabolites, thereby causing DNA damage in addition to othercontributing factors (Prokopczyk et al., 1997; Velema et al., 2002).

Since tobacco consumption is common in Pakistan and $9 \%$ of women and $36 \%$ of men are addicted to tobacco, accounting for about $32 \%$ of the total South Asian population that smoke tobacco (Moore and Tsuda, 2002). Similarly naswar consumption in Khyber
Pakhtunkhwa is very much common especially among the male Pashtun population (Afridi et al., 2000). It is possible that polymorphisms in genes encoding xenobiotic metabolizing enzymes could help in identification of susceptible individuals.

In light of the above mentioned facts, a hospitalbased case-control study was carried out to evaluate the potential role of $\mathrm{m} 1$ ( $\mathrm{T}>\mathrm{C}$, rs4646903) polymorphisms in the $C Y P 1 A 1$ gene on susceptibility to esophageal cancer patients with Pashtun ethnicity of Khyber Pakhtunkhwa Pakistan. This will help to adopt pro-active approaches for early diagnosis and preventive life style modification strategies to decrease the incidence of the disease in the target population.

\section{Materials and Methods}

\section{Sample collection}

Three milliliters of whole blood was collected from all subjects in EDTA tubes after approval from the Ethical Committee of the Department of Pharmacy, University of Peshawar (No.440, dated 17.12.2011). Informed consent and thorough interview was taken by expert in the relevant field before blood collection on a carefully designed proforma that contained information regarding age, place, occupation, socioeconomic status, cancer type and tobacco use habits etc. Study subjects comprised of 196 healthy control and 140 esophageal cancer patients between 35 and 70 years of age with Pashtun ethnicity of Khyber Pakhtunkhwa, and not less than 20 years of tobacco exposure. The patients were registered at the Institute of Radiotherapy and Nuclear Medicine (IRNUM), Peshawar, Khyber Pakhtunkhwa as per exclusion/inclusion criteria. The study period was from between June, 2012-June, 2013. All the patients were having histo-pathologically confirmed cancers.

Criteria for patients: $i$ ) Age between 35 and 70 years with Pashtun ethnicity; $i$ i) Histopathologically proven ESCC; iii) Not less than 20 years of tobacco exposure in any form.

Exclusion criteria for patients: $i$ ) Age $>70$ years; ii) Patients with non-Pashtun ethnicity

Criteria for selection of control subjects: $i$ )Normal healthy age-matched subjects of similar ethnicity with not less than 20 years of exposure to tobacco in any form, and free from esophageal cancer.

\section{DNA isolation and genotyping of CYPlAl $\mathrm{ml} T>C$ (rs4646903)}

Genomic DNA was extracted from peripheral blood leucocytes by using standard DNA Isolation kit (Purelink Genomic DNA kit Invitrogen, USA) as per supplier's recommendations. The DNA quality and quantity were estimated using a double beam spectrophotometer (Perkin Elmer series 200 system, Norwalk, USA). TheCYP1A1m1 ( $>$ C , rs4646903) polymorphism were analyzed using a highly specific Real Time Polymerase Chain Reaction (RTPCR). Light SNiP rs4646903 was purchased from TibMolbiol Germany that contained all primers and probes to run the reaction. Similarly, FastStart DNA Master Hyprobe kit required for the reaction as per supplier's 
recommendations was purchased from Roche Diagnostics Germany. Reaction was performed as per recommended guidelines with minor changes as follows. Reaction mix were prepared using Reagent Mix (1 $\mu 1)$, FastStart DNA Master $(2 \mu \mathrm{l})$, Magnesium chloride $(25 \mathrm{~mm}, 1.6 \mu \mathrm{l})$, and water $(14.4 \mu 1)$. Finally, DNA $(1 \mu 1,100-150 \mathrm{ng})$ was added to the reaction mix to make the final volume $20 \mu 1$. Thermocycler (MiniOpticon Model CFB-3120EDUUSA) conditions as per protocol were as follows:

Denaturation at $95^{\circ} \mathrm{C}$ for 10 minutes; Cycling for 45 cycles of $95^{\circ} \mathrm{C}$ for $10 \mathrm{sec}, 45^{\circ} \mathrm{C}$ for $60 \mathrm{sec}$ and $72^{\circ} \mathrm{C}$ for $15 \mathrm{sec}$; followed by melting curves analysis at $95^{\circ} \mathrm{C}$ for $10 \mathrm{sec}, 40^{\circ} \mathrm{C}$ for 2 minutes through $75^{\circ} \mathrm{C}$ for 0 sec. Duplicate samples were used as control. Melting peaks at $51-52^{\circ} \mathrm{C}$ represented wild type $(\mathrm{T} / \mathrm{T})$ allele; the one at $59-60^{\circ} \mathrm{C}$ represented variant $(\mathrm{C} / \mathrm{C})$ allele, while samples giving two peaks at $51^{\circ} \mathrm{C}$ and $59^{\circ} \mathrm{C}$ were heterozygous (T/C) allele (Figure 2).

\section{Statistical analysis}

Variables selected from the data set were age, gender, place of residence, tobacco type used (cigarette, naswar, and both cigarette and naswar), amount of tobacco used per day, age at first exposure and polymorphism in the CYP1A1 rs4646903 gene variant. Hardy-Weinberg equilibrium for control population was determined by using standard formula. Chi-square $(\chi 2)$ test was used to detect whether there were significant $(\alpha=0.05)$ differences in frequencies between cases and controls. Odds Ratios (OR) of Esophageal cancer for each polymorphism using binary logistic regression model were estimated with $95 \%$ confidence intervals (CIs), and the difference in genotype prevalence and association between case and control group were assessed independently as well as adjusted for confounding factors. Age, gender, place of residence,

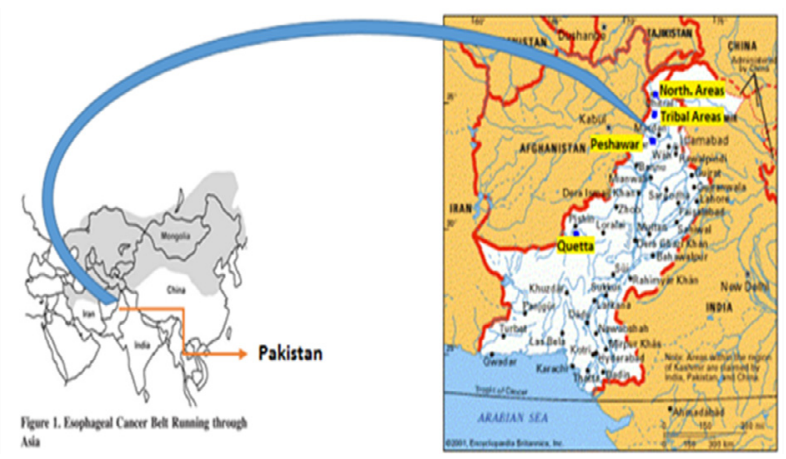

Figure 1.Possible Extension of Esophageal Cancer Belt into KP and Baluchistan Provinces ofpakistan
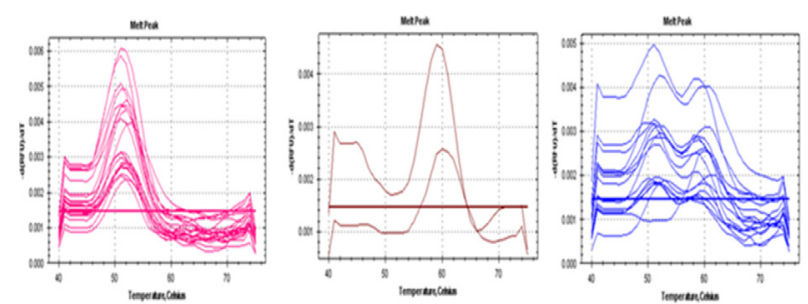

Figure 2. Melting Peak of RT-PCR: Pink, Brown and Blue Peaks Represent T/T, C/C and Heterozygou (T/C) Alleles of CYP1A1 re4646903 tobacco type used, amount of tobacco used per day and age at first exposure were included as covariates as well as all the possible genotypes studied. A probability of $\mathrm{p}<0.05$ (two sided) was considered significant to determine whether the genotype frequencies were different between the patient and control population. CYP1Al rs4646903 polymorphism was categorized into homozygous wild type and variant allele containing genotypes. Wild type was used as reference group to assess the effects of the different alleles. Analyses were performed by SPSS (Version 20.0).

\section{Results}

\section{Subject characteristics}

Demographic and other subject characteristics are shown in Table 1. Mean age of healthy subjects (controls) and esophageal cancer patients was 54.14 \pm 9.91 and $55.02 \pm 10.83$ years, respectively ( $\mathrm{t}$ test $\mathrm{p}$ value $=0.304$ ) Twenty nine percent $(29.28 \%)$ of the patients were in the age range of 41-50 years; same percentage (29.28\%) was in the age range of 51-60 years, while $28 \%$ of the patients were in the range of $60 \pm$ years of age. All the patients were male and no female patient during study period was observed. Highest incidence (27.14\%) of

\section{Table 1. Demographic Characteristics of the Subjects}

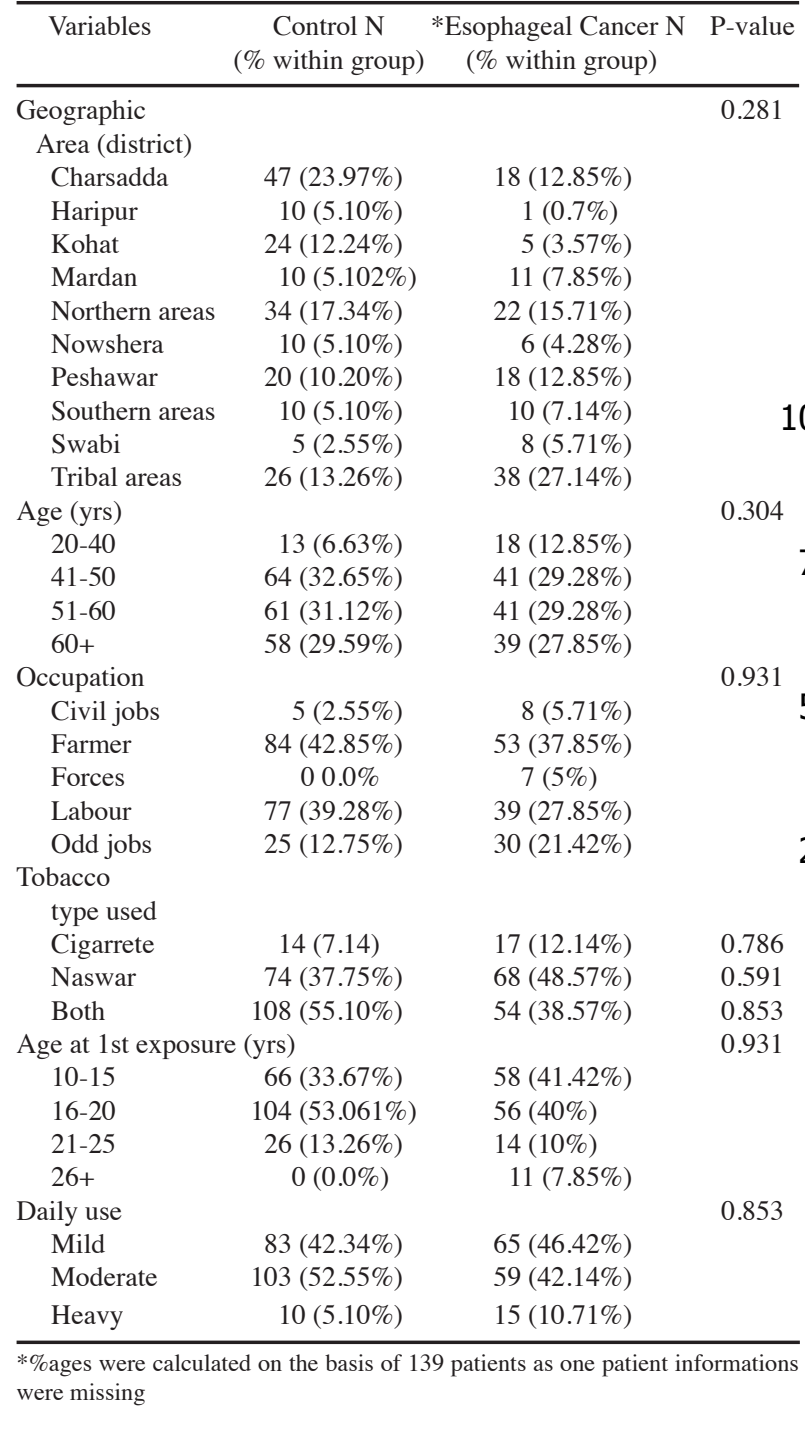


Table 2. Genotypic, Allelic and Carriage Rate Frequencies of CYP1A1 m1 (T>C,rs4646903) Gene Polymorphism in Controls $(n=196)$ and Esophageal Cancer Cases $(n=140)$.

\begin{tabular}{|c|c|c|c|c|c|c|}
\hline Genotype/allele & Case $\mathrm{N}(\%)$ & Control N(\%) & Unadjusted OR (95\% CI) & $\mathrm{p}$ & Adjusted OR (95\% CI) & $\mathrm{p}$ \\
\hline $\mathrm{T} / \mathrm{T}$ & $56(40)$ & $140(71.42)$ & Ref. & & Ref. & \\
\hline $\mathrm{C} / \mathrm{C}$ & $28(20)$ & $7(3.57)$ & $10(4.130-24.213)$ & 0 & $15.709(6.065-40.686)$ & 0 \\
\hline $\mathrm{T} / \mathrm{C}$ & $56(40)$ & $49(25)$ & $2.85(1.745-4.678)$ & 0 & $3.256(1.902-5.574)$ & 0 \\
\hline$* \mathrm{~T}$ allele & $168(60)$ & $329(83.92)$ & Ref. & & & \\
\hline$*$ C allele & $112(40)$ & $63(16.07)$ & $6.811(3.706-12.518)$ & 0 & $9.967(5.229-18.997)$ & 0 \\
\hline \multicolumn{7}{|c|}{ Allele carriage rate } \\
\hline $\mathrm{T}(+)$ & $112(80)$ & $189(96.42)$ & Ref. & & & \\
\hline $\mathrm{T}(-)$ & $28(20)$ & $7(3.57)$ & $6.811(2.880-16.107)$ & 0 & $9.967(4.003-24.815)$ & 0 \\
\hline $\mathrm{C}(+)$ & $84(60)$ & $56(28.57)$ & Ref. & & & \\
\hline $\mathrm{C}(-)$ & $56(40)$ & $140(71.42)$ & $.267(.169-.422)$ & 0 & & \\
\hline
\end{tabular}

EC was observed in tribal areas, followed by Northern areas (15.71\%), Charsadda and Peshawar (12.85\% each). To estimate the socioeconomic status occupation of the subjects was observed. Highest incidence $(37.85 \%)$ was observed in farmers followed by laborer $(27.85 \%)$ and other odd jobs (21.42\%). Several aspects of tobacco use habit were noted including type of tobacco product used, age at $1^{\text {st }}$ exposure and amount of tobacco used per day. Cases and control were having similar tobacco habits $(\mathrm{t}$ test $p$ values $=0.786,0.591$ and 0.853 for types of tobacco used (cigarettes, naswar, both types), age at first exposure, and amount of tobacco used respectively. Majority $(48.57 \%)$ of the patients were naswar addicts followed by combined use of naswar and cigarettes $(38.57 \%)$. Only $12.14 \%$ were addicted to cigarettes alone. Forty one percent patients started tobacco at the age of 10-15 years, while $40 \%$ started at the age of between 16-20 years, with mean age of 17 years. Subjects were divided into three categories on the basis of amount of tobacco used per day. Mild users were those taking less than 0.5 packs to 1.5 packs of either naswar or cigarettes alone or in combination. Moderate users were those taking more than 1.5 packs to 3 packs of either naswar or cigarettes alone or in combination. Heavy users were those using more than 4 packs of either naswar or cigarettes alone or in combination. Majority $(46.42 \%)$ of the patients were mild users followed by moderate users $(42.14 \%)$. Only about $11 \%$ were heavy users.

\section{Association with susceptibility to esophageal cancer}

The allele frequencies and genotype of CYP1A1 rs4646903 polymorphism among controls were not departure from Hardy-Weinberg equilibrium. The distributions of genotypes of CYPIA1 rs4646903 were significantly different between the cases and controls groups (Pearson chi Square $\chi 2_{0.05,2}=0.000, \mathrm{p}>0.05$ ) (Table $2)$. The prevalence of CC and TC genotypes was more in cases (20\% and $40 \%$ respectively) when compared to controls $(3.57 \%$ and $25 \%$ ). Homozygous variant type (C/C) had a 10-fold increased risk for EC, compared with wild genotype (T/T) (OR: 10 (4.130-24.213)); while heterozygous variant $(\mathrm{T} / \mathrm{C})$ had almost 3 -fold increased risk for EC, compared with wild genotype (T/T)(OR: $2.85(1.745-4.678))$ at $95 \% \mathrm{CI}$. The risk showed a further increase (5-fold in case of $\mathrm{C} / \mathrm{C}$ allele) when the data were adjusted for age, gender, place of residence, tobacco type used, amount of tobacco used per day and age at first exposure $(\mathrm{OR}=15.709$ (6.065-40.686) and 3.256 (1.9025.574 respectively).

\section{Discussion}

The ubiquitous family of enzymes cytochrome P450 plays an important role in the metabolic activation of major classes of tobacco related carcinogens. These include phase I enzyme CYP1A1 that is responsible for the activation of benzo[a]pyrene and nitrosamines thus affecting the metabolism of the environmental carcinogens and altering the susceptibility of esophageal cancer. Variation in CYP1A1 gene may alter function of its enzymes in such a way that toxicity of the extraneous stimulating factors that directly influence tissues may be enhanced, thus increasing susceptibility to cancer. Two major relevant polymorphic sites of the CYP1A1 gene have been associated with several types of cancer. One site (known as Msp1 orrs4646903) is located in the 3'-flanking region of the gene ( $\mathrm{T} 6235 \mathrm{C}$ position) in which the presence of $\mathrm{C}$ has been considered important risk factor. While the other (known as Ile/Val) (A4889G) is located at codon 462 that alters the protein structure by replacing an isoleucine for a valine, making the carriers more susceptible to some tobacco-associated cancers (Wang et al., 2002b). A casecontrol study with molecular epidemiology methods was designed to analyze the relationship between CYP1A1 gene variant rs4646903 and esophageal cancer risk. Our data showed that the replacement from $\mathrm{T}$ to $\mathrm{C}$ at this site can significantly increase the risk of esophageal cancer. Variant allele (CC) ten times increases the risk of EC in Pashtun population while heterozygous allele (T/C) increases the risk about three fold. After adjusting for age, gender, place of residence, tobacco type used, amount of tobacco used per day and age at first exposure the risk further increases (Adjusted $\mathrm{OR}=15.709$ and 3.256 respectively). This shows that the above mentioned environmental factors also significantly contribute towards the risk of developing EC. The gender, place of residence and naswar use in this study are among the factors that increase the risk. This confirms various studies that have previously reported the high prevalence of EC in Khyber Pakhtunkhwa and other Pashtun population areas (Jamal et al., 1997; Afridi et al., 2000; Shahid Jamal 2010). Remarkable proportional differences have been observed 
between male and female regarding occurrence of EC. Studies have reported differences up to 1:5 between females and males (Cook et al., 2009; Hajmanoochehri et al., 2013). Interestingly we have not observed any female patient of EC in our one year time period. The occurrence of EC increases with age, with incidence rates peaking at 70 years of age. The peak age in majority of patients with EC is over the age of 50 years. In this study almost constant proportion has been observed in patients above 40 years up to 70 years of age. Associated risk factors for $\mathrm{EC}$ are well known. Risk factors previously reported for ESCC include tobacco use, alcohol consumption, and under-nutrition. Low socioeconomic status is associated with under-nutrition in developing countries. As a risk factor, under-nutrition includes low intake of fresh fruits and vegetables and low intake of micronutrients such as vitamin A, C, E, riboflavin, zinc and selenium. Studies have demonstrated a reduced risk of EC associated with regular intake of fresh fruits and vegetables with former to be more beneficial than latter (Yang et al., 2005; Yamaji et al., 2008; Mao et al., 2012; Song 2013). Same finding were observed over here. Almost all patients were of low socioeconomic status that was evident from their life long profession. High prevalence in certain geographical areas has been reported for EC as discussed previously. Our observations were consistent with those studies and high prevalence of EC in areas adjacent to Afghanistan border (Tribal areas, Northern areas, Charsadda and Peshawar) further provides yet another evidence for the hypothesis that Pashtun population areas of Pakistan are an extension of 'Asian cancer belt' (Jamal 2010). Regarding tobacco habits our study showed consistent results with previous studies. Almost eighty percent of the patients started tobacco at 10-20 years of age. Similarly, almost eighty percent of the patients were mild to moderate users. This shows that routine doses of tobacco play important role in the incidence of EC. Naswar was observed to be the main tobacco product consumed by the patients. Its association with EC has been shown previously (Afridi et al., 2000). This may be because naswar is a smokeless tobacco product and is swallowed with the saliva that comes in direct contact with esophagus. Naswar contains various carcinogenic species especially TSNAs, and also has a basic pH of more than 8 (Zakiullah et al., 2012). Basic $\mathrm{pH}$ has the same effect as that of alcohol in making carcinogenic species more soluble, leading to their more efficient absorption into esophageal epithelium. That may explain that why naswar is an important risk factor for the development of EC.

Polymorphism studies at rs4646903 site have shown different results. Some have reported association (Guo et al., 2005), while others have shown no or weak association (Wu et al., 2002; Casson et al., 2003; Wang et al., 2003) with EC. This shows that factors such as ethnicity, study population, different environmental exposure and sample size may affect the results. Therefore, large population based studies should be designed in specific ethnic groups exposed to similar risk factors to analyze the relationship of CYP1A1 rs4646903 genetic polymorphism and esophageal cancer.

In conclusion, our study suggests that the CYP1A1 rs4646903 gene variations may be associated with the risk of esophageal cancer. However, large population based epidemiologic studies should be conducted in future to consider interactions between genetic polymorphisms and exposure to environmental carcinogens to make the tests results more objective and reliable. Conclusion and recommendations. Despite high prevalence in Khyber Pakhtunkhwa the precise etiologic factors for EC have not been elucidated. As discussed in the risk factors, reducing consumption of tobacco (especially naswar in Pashtun population) and more consumption of fruits and vegetables must be regarded as the primary preventive strategies. Similarly, projects should be designed by governmental agencies to screen for genetically susceptible individuals and awareness regarding genetic susceptibility and environmental risk factors be generated in general public. Also, further research is recommended to understand the precise etiology and epidemiology of EC to prevent the general public from this fatal and costly disease.

\section{Acknowledgements}

We acknowledge the blood samples of cases given by the Institute of Radiotherapy and Nuclear Medicine (IRNUM), Peshawar. We also wish to acknowledge Mr. Waheed-ur-Rahman, Mr. Zahid Ali and Mr. Zahir Shah, who collected the control blood samples from different districts of Khyber Pakhtunkhwa, and last but not the least we also acknowledge our valued patients and control individuals who participated in the research through their blood sample donation.

\section{References}

Abbas M, Srivastava K, Imran M, Banerjee M (2014). Association of CYP1Al gene variants rs4646903 (T>C) and $\mathrm{rs} 1048943(\mathrm{~A}>\mathrm{G})$ with cervical cancer in a north indian population. Eur J Obstet Gynecol Reprod Biol. 176, 68-74.

Afridi SP, Khan A, Waheed I (2000). High risk factors in patient with carcinoma esophagus. J Coll Physicians Surg Pak, 10, 368-70.

Amani F, Ahari SS, Akhghari L (2013). Epidemiology of esophageal cancer in Ardabil province during 2003-2011. Asian Pac J Cancer Prev, 14, 4177-80.

Badar F, Anwar N, Mahmood S (2005). Geographical variation in the epidemiology of esophageal cancer in pakistan. Asian Pac J Cancer Prev, 6, 139-42.

Blot W, McLaughlin J, Fraumeni JF (2006). Esophageal Cancer. In Cancer Epidemiology and Prevention Edited by: Schottenfeld D, Fraumeni J. New York: Oxford University Press, 697-706.

Castellsagué X, Munoz N, De Stefani E, et al (1999). Independent and joint effects of tobacco smoking and alcohol drinking on the risk of esophageal cancer in men and women. Int $J$ Cancer, 82, 657-64.

Cook MB, Dawsey SM, Freedman ND, et al (2009). Sex disparities in cancer incidence by period and age. Cancer Epidemiol Biomarkers Prev, 18, 1174-82.

Cortes GR, Villasenor CR (1997). Cancer deesofago. Rev Gastroenterol Mex, 62, 149-59.

D'Errico A, Taioli E, Xhen X, Vineis P (1996). Genetic metabolic polymorphisms and the risk of cancer: a review of the literature. Biomarkers, 1, 149-73.

Eslick G (2009). Epidemiology of esophageal cancer. 
Gastroenterol Clin N Am, 38, 17-25.

Jamal S, Ahmad M, Khan AH, et al (1997). Carcinoma esophagus-a clinicopathological study of 50 cases at Armed Forces Institute of Pathology, Rawalpindi, Pakistan. $J$ Pathol, 8, 24-8.

Hajmanoochehri F, Mohammadi N, Nasirian N, Hosseinkhani M (2013). Patho-epidemiological features of esophageal and gastric cancers in an endemic region: a 20-year retrospective study. Asian Pac J Cancer Prev, 14, 3491-7.

Jamal S (2010). Esophageal cancer in Pakistan-is it really extension of Asian cancer belt? Pak Arm For Med J, 2.

Jemal A, Bray F, Center MM, et al (2011). Global cancer statistics. CA Cancer J Clin, 61, 69-90.

Kollarova H, Machova L,Horakova D, et al (2007). Epidemiology of esophageal cancer-an overview article. Biomed Pap Med Fac Univ Palacky Olomouc Czech Repub, 151, 17-28.

Mao WM, Zheng WH, Ling ZQ (2012). Epidemiologic risk factors for esophageal cancer development. Asian Pac J Cancer Prev, 12, 2461-6.

Moore MA, Tsuda H (2002). Smoking-related research in the Asian-pacific countries. Asian Pac J Cancer Prev, 3, 291304.

Olshan AF, Weissler MC, Watson MA, Bell DA (2000). GSTM1, GSTT1, GSTP1, CYP1A1, and NAT1 polymorphisms, tobacco use, and the risk of head and neck cancer. Cancer Epidemiol Biomark Prev, 9, 185-91.

Prokopczyk B, Cox JE, Hoffmann D, Waggoner SE (1997). Identification of tobacco specific carcinogen in the cervical mucus of smokers and non-smokers. J Natl Cancer Inst, 89, 868-73.

Sabitha K, Reddy MV, Jamil K (2010). Smoking related risk involved in individuals carrying genetic variants of CYP1A1 gene in head and neck cancer. Cancer Epidemiol, 34, 587-92.

Sharma MK, Gour N,Pandey A, Wallia D (2011). Epidemiological study of risk factors for oral, laryngeal and esophageal cancers at a tertiary care hospital in India. Asian Pac J Cancer Prev, 12, 1215-18.

Weng Y, Fei B, He P, Cai M (2012).Glutathione-s-transferase t1 polymorphism is associated with esophageal cancer risk in chinese han population. Asian Pac J Cancer Prev, 13, 4403-07.

Taskiran C,Aktas D, Yigit-Celik N, et al (2006). CYP1A1 gene polymorphism as a risk factor for cervical intra epithelial neoplasia and invasive cervical cancer. Gynecol Oncol, 101, 503-6.

Velema JP, Ferrera A, Figueroa M, et al (2002). Burning wood in the kitchen increases the risk of cervical neoplasia in HPV-infected women in honduras. Int J Cancer, 97, 536-41.

Xia L, Gao J, Liu Y, Wu K, (2013). Significant association between CYP1A1 T3801C polymorphism and cervical neoplasia risk: a systematic review and meta-analysis. Tumor Biol, 34, 223-30.

Yamaji T, Inoue M, Sasazuki S, et al (2008). Fruit and vegetable consumption and squamous cell carcinoma of the esophagus in Japan: the JPHC study. japan public health center-based prospective study group. Int J Cancer, 123, 1935-40.

Yang CX, Wang HY, Wang ZM et al (2005). Risk factors for esophageal cancer: a case control study in southwestern china. Asian Pac J Cancer Prev, 6, 48-53.

Yi SM, Li GY (2012). Null genotype of GSTT1 contributes to esophageal cancer risk in Asian populations: evidence from a meta-analysis. Asian Pacific J Cancer Prev, 13, 4967-71.

Yun YX, Wang YP, Wang P, et al (2013). CYP1A1 genetic polymorphisms and risk for esophageal cancer: a casecontrol study in central China. Asian Pac J Cancer Prev, 14, 6507-12.

Zakiullah, Saeed M, Muhammad N, et al (2012). Assessment of potential toxicity of smokeless tobacco product (naswar)available on the pakistani market. Tob.Control, 21, 396-401. Song QK, Zhao L, Li J, et al, (2013). Adverse effects of preserved vegetables on squamous cell carcinoma of esophagus and precancer lesions in a high risk area. Asian Pac J Cancer Prev, 14, 659-63. 